\title{
THE THERMISTOR PROBLEM FOR CONDUCTIVITY WHICH VANISHES AT LARGE TEMPERATURE
}

\author{
BY \\ XINFU CHEN AND AVNER FRIEDMAN \\ University of Minnesota, Minneapolis, Minnesota
}

\begin{abstract}
The thermistor problem is modeled as a coupled system of nonlinear elliptic equations. When the conductivity coefficient $\sigma(u)$ vanishes $(u=$ temperature) one of the equations becomes degenerate; this situation is considered in the present paper. We establish the existence of a weak solution and, under some special Dirichlet and Neumann boundary conditions, analyze the structure of the set $\{\sigma(u)=0\}$ and also prove uniqueness.
\end{abstract}

1. Introduction. A thermistor is an electric circuit device made of ceramic material whose electrical conductivity $\sigma(u)$ decreases several orders of magnitude as the temperature $u$ increases beyond a critical temperature $u^{*}$. Denote by $\Omega$ the domain in $\mathbb{R}^{N}$ occupied by the thermistor, by $\varphi$ the electric potential, and by $k=k(u)$ the thermal conductivity. Then

$$
\begin{aligned}
& J=\text { electric current density }=-\sigma(u) \nabla \varphi, \\
& q=\text { heat flux }=-k(u) \nabla u, \quad E=-\nabla \varphi=\text { electric field. }
\end{aligned}
$$

The conservation of current $\nabla \cdot J=0$ and of energy $\nabla \cdot q=J \cdot E$ can then be written in the form

$$
\nabla(\sigma(u) \nabla \varphi)=0 \quad \text { in } \Omega
$$

and

$$
\nabla(k(u) \nabla u)=-\sigma(u) \nabla \varphi \cdot \nabla \varphi=-\nabla(\sigma(u) \varphi \nabla \varphi) \quad \text { in } \Omega
$$

where equation (1.1) was used in deriving the last equation in $\left(1.1_{\mathrm{a}}\right)$. Since $k(u)$ varies only slightly with $u$, we shall assume in the sequel that $k(u) \equiv 1$; all our results, however, extend to general $k=k(u)$. Equation $\left(1.1_{\mathrm{a}}\right)$ then becomes

$$
\nabla(\nabla u+\sigma(u) \varphi \nabla \varphi)=0 \text { in } \Omega,
$$

or

$$
\nabla^{2} u+\sigma(u)|\nabla \varphi|^{2}=0 \quad \text { in } \Omega .
$$

For the physical background of the thermistor problem and some explicit solutions we refer to [1], [9], [10], [11], and the references therein. There has been recent 
mathematical interest in the problem in case $\sigma(u)$ is uniformly positive; see [2], [3], [4], [7], [8]. Cimatti and Prodi in [2] and Cimatti in [3] considered the Dirichlet boundary conditions for both $\varphi$ and $u$ and proved existence of a solution. In [4] Cimatti extended the existence result to the case where

$$
\begin{gathered}
\varphi=\varphi^{0}, \quad u=u^{0} \quad \text { on } \Gamma_{D}, \quad \Gamma_{D} \subset \partial \Omega, \\
\frac{\partial \varphi}{\partial n}=0, \quad \frac{\partial u}{\partial n}=0 \quad \text { on } \Gamma_{N}=\partial \Omega \backslash \bar{\Gamma}_{D} .
\end{gathered}
$$

An important observation by Diesselhorst [5] that the function

$$
\psi=\frac{1}{2} \varphi^{2}+\int_{0}^{u} \frac{d s}{\sigma(s)}
$$

satisfies the equation

$$
\nabla(\sigma(u) \nabla \psi)=0 \quad \text { in } \Omega,
$$

plays a crucial role in the papers [3], [4].

In the special case

$$
\begin{aligned}
& \Gamma_{D}=\Gamma_{1} \cup \Gamma_{2}, \quad \varphi=\varphi_{i}, \quad u=u_{i} \text { on } \Gamma_{i}, \\
& \varphi_{i} \text { and } u_{i} \text { are constants and } \bar{\Gamma}_{1} \cap \bar{\Gamma}_{2}=\varnothing, \\
& \frac{\partial \varphi}{\partial n}=0, \quad \frac{\partial u}{\partial n}=0 \quad \text { on } \Gamma_{N} \equiv \partial \Omega \backslash \bar{\Gamma}_{D},
\end{aligned}
$$

Cimatti also proved uniqueness; but, in general, uniqueness is still an open problem.

More recently Howison, Rodrigues, and Shillor [8] have extended the existence result to more general boundary conditions, such as

$$
\begin{gathered}
\varphi=\varphi^{0} \quad \text { on } \Gamma_{D}^{1}, \quad \frac{\partial \varphi}{\partial n}=0 \quad \text { on } \partial \Omega \backslash \overline{\Gamma_{D}^{1}}, \\
u=u^{0} \quad \text { on } \Gamma_{D}^{2}, \quad \frac{\partial u}{\partial n}+\gamma u=g_{0} \quad \text { on } \partial \Omega \backslash \overline{\Gamma_{D}^{2}} .
\end{gathered}
$$

In this paper we are interested in the case where $\sigma(u)$ vanishes for large $u$, i.e.,

$$
\sigma(u)>0 \quad \text { if } u<u^{*}, \quad \sigma(u)=0 \quad \text { if } u>u^{*}
$$

for some constant $u^{*}$. This provides a good approximation to the actual engineering model of thermistors, whereby the conductivity $\sigma(u)$ drops to nearly 0 beyond some critical temperature $u^{*}$. We shall be working with the boundary conditions (1.4).

In Sec. 2 we approximate $\sigma(u)$ by a family of uniformly positive functions $\sigma_{\varepsilon}(u)$ and review the existence proof of a solution $\left(\varphi_{\varepsilon}, u_{\varepsilon}\right)$. We also derive a priori estimates independent of $\varepsilon$. In particular, we prove that

$$
\int_{\Omega}\left|\nabla \sigma_{\varepsilon}\left(u_{\varepsilon}\right)\right|^{\beta} \leq C \quad \text { if } \frac{1}{2(1-\alpha)}<\beta \leq 1
$$

provided

$$
\left|\sigma_{\varepsilon}^{\prime}(u)\right| \leq C\left(\left|u^{*}-u\right|^{-\alpha}+1\right), \quad \alpha \in\left(0, \frac{1}{2}\right) .
$$

In Sec. 3 we define the concept of a weak solution $(\varphi, u)$ for (1.1), (1.2), (1.4) and prove that a subsequence of $\left(\varphi_{\varepsilon}, u_{\varepsilon}\right)$ converges to a weak solution. 
In Sec. 4 we specialize to the boundary conditions (1.7) and prove additional properties of $(\varphi, u)$. In particular, $\sigma(u(x))$ is a continuous function, the level surface

$$
S=\{x \in \Omega ; \sigma(u(x))=0\}
$$

is piecewise analytic (analytic if $N=2$ ), and $\varphi(x)$ is continuous in $\Omega \backslash S$ with jump discontinuity across $S$. We also prove uniqueness.

Finally, in Sec. 5 we consider special solutions with boundary conditions of the form (1.8) for which the set $\{\sigma(u(x))=0\}$ has nonempty interior.

2. The approximating problem. For simplicity we take $u^{*}=0$ in (1.9). We shall assume that

$$
\begin{array}{ll}
0<\sigma(u)<M & \text { if } u<0, \\
\sigma(u)=0 \quad \text { if } u>0, & \sigma \in C^{0}(-\infty, \infty),
\end{array}
$$

and

$$
\begin{gathered}
\sigma \in C^{1}(-\infty, 0), \\
\left|\sigma^{\prime}(u)\right| \leq M_{0}\left(1+|u|^{-\alpha}\right) \quad \text { if } u<0, \text { for some } \alpha \in\left(0, \frac{1}{2}\right)
\end{gathered}
$$

this implies that for $u<0$,

$$
\sigma(u) \leq c|u|^{\alpha^{\prime}}, \quad \alpha^{\prime}=(1-\alpha) \in\left(\frac{1}{2}, 1\right) .
$$

We introduce a family of smooth functions $\sigma_{\varepsilon}(u) \quad(0<\varepsilon<1)$ which approximate $\sigma(u)$ as $\varepsilon \rightarrow 0$, each uniformly positive:

$$
\begin{array}{ll}
\varepsilon \leq \sigma_{\varepsilon}(u) \leq 2 M \quad \forall u, \\
\sigma_{\varepsilon}(u)=\varepsilon \quad \text { if } u>0, \quad \sigma_{\varepsilon} \in C^{\infty}(-\infty, \infty),
\end{array}
$$

and

$$
\sigma_{\varepsilon}(u) \rightarrow \sigma(u) \text { as } \varepsilon \rightarrow 0 \text {, uniformly in } u \text { in bounded intervals. }
$$

We also take the $\sigma_{\varepsilon}$ to satisfy

$$
\left|\sigma_{\varepsilon}^{\prime}(u)\right| \leq 2 M_{0}\left(1+|u|^{-\alpha}\right) \quad \forall u,
$$

with the same $\alpha$ as in (2.2).

We assume that $\partial \Omega$ is piecewise $C^{1+\delta}$ for some $0<\delta<1$, and that $\partial \Gamma_{D}$ is piecewise $C^{1+\delta}$. We also assume that the boundary data $\varphi^{0}, u^{0}$ can be extended into $\Omega$ so that

$$
\begin{array}{ll}
\left\|\varphi^{0}\right\|_{L^{\infty}(\Omega)}<\infty, & \int_{\Omega}\left|\nabla \varphi^{0}\right|^{2}<\infty, \\
\left\|u^{0}\right\|_{L^{\infty}(\Omega)}<\infty, & \int_{\Omega}\left|\nabla u^{0}\right|^{2}<\infty .
\end{array}
$$

Finally we assume that $\left.u^{0}\right|_{\Gamma_{D}}$ is smaller than the critical temperature $u^{*}(=0)$, i.e.,

$$
\left.u^{0}\right|_{\Gamma_{D}} \leq-c_{*}<0
$$


If we choose the extension of $\left.u^{0}\right|_{\Gamma_{D}}$ to be such that

$$
\Delta u^{0}=0 \quad \text { in } \Omega, \quad \frac{\partial u^{0}}{\partial n}=0 \quad \text { on } \Gamma_{N},
$$

then (2.7) is of course again satisfied and further, by the maximum principle,

$$
u^{0} \leq-c_{*}<0 \text { in } \bar{\Omega} \text {. }
$$

Consider the elliptic system

$$
\begin{aligned}
& \nabla\left(\sigma_{\varepsilon}\left(u_{\varepsilon}\right) \nabla \varphi_{\varepsilon}\right)=0 \text { in } \Omega, \\
& \Delta u_{\varepsilon}+\sigma_{\varepsilon}\left(u_{\varepsilon}\right)\left|\nabla \varphi_{\varepsilon}\right|^{2}=0 \text { in } \Omega \text {, } \\
& \varphi_{\varepsilon}=\varphi^{0} \quad \text { on } \Gamma_{D}, \quad \frac{\partial \varphi_{\varepsilon}}{\partial n}=0 \text { on } \Gamma_{N} \text {, } \\
& u_{\varepsilon}=u^{0} \quad \text { on } \Gamma_{D}, \quad \frac{\partial u_{\varepsilon}}{\partial n}=0 \text { on } \Gamma_{N},
\end{aligned}
$$

where $\Gamma_{N}=\partial \Omega \backslash \bar{\Gamma}_{D}$.

Lemma 2.1. There exists a solution $\left(\varphi_{\varepsilon}, u_{\varepsilon}\right)$ of $(2.11)-(2.14)$ in $L^{\infty}(\Omega) \cap H^{1}(\Omega)$, having the following properties:

(i) $\varphi_{\varepsilon}$ and $u_{\varepsilon}$ belong to $C^{\infty}(\Omega)$, and

(ii) if $\partial \Omega \in C^{m+\delta}, \varphi^{0} \in C^{m+\delta}\left(\Gamma_{D}\right), u^{0} \in C^{m+\delta}\left(\Gamma_{D}\right)$ then $\varphi_{\varepsilon}$ and $u_{\varepsilon}$ belong to $C^{m+\delta}\left(\bar{\Omega} \backslash\left(\bar{\Gamma}_{D} \cap \bar{\Gamma}_{N}\right)\right) \quad(m=1,2, \ldots)$.

The proof given below is essentially due to Cimatti [4].

Proof. Introducing the change of variables

$$
\psi_{\varepsilon}=\frac{1}{2} \varphi_{\varepsilon}^{2}+\int_{-1}^{u_{\varepsilon}} \frac{d t}{\sigma_{\varepsilon}(t)},
$$

we can rewrite $(2.11)-(2.14)$ in the form

$$
\begin{aligned}
& \nabla\left(a_{\varepsilon}\left(\psi_{\varepsilon}-\frac{1}{2} \varphi_{\varepsilon}^{2}\right) \nabla \varphi_{\varepsilon}\right)=0 \text { in } \Omega, \\
& \nabla\left(a_{\varepsilon}\left(\psi_{\varepsilon}-\frac{1}{2} \varphi_{\varepsilon}^{2}\right) \nabla \psi_{\varepsilon}\right)=0 \text { in } \Omega, \\
& \varphi_{\varepsilon}=\varphi^{0} \text { on } \Gamma_{D}, \quad \frac{\partial \varphi_{\varepsilon}}{\partial n}=0 \text { on } \Gamma_{N}, \\
& \psi_{\varepsilon}=\psi_{\varepsilon}^{0} \equiv \frac{1}{2} \varphi^{02}+\int_{-1}^{u^{0}} \frac{d t}{\sigma_{\varepsilon}(t)} \text { on } \Gamma_{D}, \quad \frac{\partial \psi_{\varepsilon}}{\partial n}=0 \text { on } \Gamma_{N},
\end{aligned}
$$

where

$$
a_{\varepsilon}(s)=\sigma_{\varepsilon}\left(F_{\varepsilon}^{-1}(s)\right)
$$

and $u=F_{\varepsilon}^{-1}(s)$ is the inverse function of

$$
s=F_{\varepsilon}(u)=\int_{-1}^{u} \frac{d t}{\sigma_{\varepsilon}(t)} .
$$


Define a mapping $T: L^{2}(\Omega) \times L^{2}(\Omega) \rightarrow H^{1}(\Omega) \times H^{1}(\Omega)$ by $(\varphi, \psi)=T(\tilde{\varphi}, \tilde{\psi})$ where $(\varphi, \psi)$ is the solution of $(2.16)-(2.19)$ with $a_{\varepsilon}\left(\psi_{\varepsilon}-\varphi_{\varepsilon}^{2} / 2\right)$ replaced by $a_{\varepsilon}\left(\tilde{\psi}_{\varepsilon}-\tilde{\varphi}_{\varepsilon}^{2} / 2\right)$. By the standard theory for elliptic equations in divergence form we know that $T(\tilde{\varphi}, \tilde{\psi})$ is well defined and

$$
\|\varphi\|_{L^{\infty}(\Omega)} \leq\left\|\varphi^{0}\right\|_{L^{\infty}(\Omega)}, \quad\|\psi\|_{L^{\infty}(\Omega)} \leq\left\|\psi_{\varepsilon}^{0}\right\|_{L^{\infty}(\Omega)} .
$$

Further, multiplying the equations for $\varphi$ and $\psi$ by $\varphi-\varphi^{0}$ and $\psi-\psi_{\varepsilon}^{0}$ respectively, and integrating over $\Omega$, we find that

$$
\left\|\varphi_{\varepsilon}\right\|_{H^{1}(\Omega)} \leq C_{\varepsilon}, \quad\left\|\psi_{\varepsilon}\right\|_{H^{1}(\Omega)} \leq C_{\varepsilon},
$$

where $C_{\varepsilon}$ is a constant independent of $\tilde{\varphi}, \tilde{\psi}$. It follows that $T$ maps $L^{2}(\Omega) \times L^{2}(\Omega)$ into a compact set, and one can easily verify that $T$ is also continuous. Hence, by Schauder's fixed point theorem, $T$ has a fixed point $\left(\varphi_{\varepsilon}, \psi_{\varepsilon}\right)$, which yields via (2.15) a solution $\left(\varphi_{\varepsilon}, u_{\varepsilon}\right)$ to $(2.11)-(2.14)$. By elliptic estimates (see, for instance, [6]) we have that $\varphi_{\varepsilon}, \psi_{\varepsilon}$ belong to $C^{\rho}(\Omega)$ for some $\rho \in(0,1)$ and therefore $u_{\varepsilon}$ is also in the same $C^{\rho}$ class. Using this fact we can deduce from (2.16), (2.17) that $\varphi_{\varepsilon}, \psi_{\varepsilon}$ belong to $C^{1+\rho}(\Omega)$, and then also $u_{\varepsilon} \in C^{1+\rho}(\Omega)$. By the same bootstrap argument one can proceed to show that $\varphi_{\varepsilon}$ and $u_{\varepsilon}$ belong to $C^{\infty}(\Omega)$. The proof of the last assertion of the lemma is obtained by a similar argument.

REMARK 2.1. The assumption (2.5) was not used in the proof of Lemma 2.1.

LemMA 2.2. The solution $\left(\varphi_{\varepsilon}, u_{\varepsilon}\right)$ satisfies:

$$
\begin{aligned}
& \left\|\varphi_{\varepsilon}\right\|_{L^{\infty}(\Omega)}+\left\|u_{\varepsilon}\right\|_{L^{\infty}(\Omega)} \leq C, \\
& \int_{\Omega}\left|\nabla u_{\varepsilon}\right|^{2} \leq C, \\
& \int_{\Omega} \sigma_{\varepsilon}\left(u_{\varepsilon}\right)\left|\nabla \varphi_{\varepsilon}\right|^{2} \leq C, \\
& \int_{\Omega}\left|\nabla \sigma_{\varepsilon}^{\beta}\left(u_{\varepsilon}\right)\right|^{2} \leq C_{\beta} \quad \text { for any } \frac{1}{2(1-\alpha)}<\beta \leq 1,
\end{aligned}
$$

where $C, C_{\beta}$ are constants independent of $\varepsilon$.

Proof. The estimate (2.23) follows from the proof of Lemma 2.1 since $\left\|\psi_{\varepsilon}^{0}\right\|_{L^{\infty}} \leq$ $C_{0}$, where $C_{0}$ is independent of $\varepsilon$ (by (2.10) and (2.3), (2.4)). Next,

$$
\begin{aligned}
\int_{\Omega} \sigma_{\varepsilon}\left(u_{\varepsilon}\right)\left|\nabla \varphi_{\varepsilon}\right|^{2} & =\inf _{\varphi \in H^{1}(\Omega), \varphi=\varphi^{0} \text { on } \Gamma_{D}} \int_{\Omega} \sigma_{\varepsilon}\left(u_{\varepsilon}\right)|\nabla \varphi|^{2} \\
& \leq\left\|\sigma_{\varepsilon}\left(u_{\varepsilon}\right)\right\|_{L^{\infty}} \int_{\Omega}\left|\nabla \varphi^{0}\right|^{2} \leq C
\end{aligned}
$$

since $0<\sigma_{\varepsilon}(t) \leq 2 M$; thus $(2.25)$ holds.

To prove (2.24) and (2.26) we multiply both sides of (2.12) by $f\left(u_{\varepsilon}\right)-f\left(u^{0}\right)$ and integrate over $\Omega$. After integrating by parts we get

$$
\int_{\Omega} f^{\prime}\left(u_{\varepsilon}\right)\left|\nabla u_{\varepsilon}\right|^{2} \leq \int_{\Omega} f^{\prime}\left(u^{0}\right) \nabla u_{\varepsilon} \cdot \nabla u^{0}+\int_{\Omega}\left(f\left(u_{\varepsilon}\right)-f\left(u^{0}\right)\right) \sigma_{\varepsilon}\left(u_{\varepsilon}\right)\left|\nabla \varphi_{\varepsilon}\right|^{2} .
$$


Using the Schwarz inequality on the first integral on the right-hand side, we obtain

$$
\int_{\Omega} f^{\prime}\left(u_{\varepsilon}\right)\left|\nabla u_{\varepsilon}\right|^{2} \leq \int_{\Omega} \frac{f^{\prime}\left(u^{0}\right)^{2}}{f^{\prime}\left(u_{\varepsilon}\right)}\left|\nabla u^{0}\right|^{2}+C\left\|f\left(u_{\varepsilon}\right)-f\left(u^{0}\right)\right\|_{L^{\infty}(\Omega)},
$$

where $(2.25)$ was used.

Taking $f(s)=s,(2.24)$ follows. To prove $(2.26)$ we take

$$
f(s)=\int_{0}^{s}\left[\left|\frac{d}{d s} \sigma_{\varepsilon}^{\beta}(s)\right|^{2}+1\right] d s ;
$$

the condition (2.5) implies that the integrand is integrable. We then get from (2.27)

$$
\int_{\Omega}\left|\nabla \sigma_{\varepsilon}^{\beta}\left(u_{\varepsilon}\right)\right|^{2} \leq \int_{\Omega} \frac{f^{\prime}\left(u^{0}\right)^{2}}{f^{\prime}\left(u_{\varepsilon}\right)}\left|\nabla u^{0}\right|^{2}+C,
$$

and $\left|f^{\prime}\left(u^{0}\right)\right| \leq C$ since $u^{0} \leq-c_{*}<0$. Since $f^{\prime}\left(u_{\varepsilon}\right) \geq 1$, the assertion (2.26) follows.

3. Existence of a weak solution. Consider (1.1), (1.3). Using the formula $\sigma(u) \nabla \varphi$ $=\nabla(\sigma(u) \varphi)-\varphi \nabla \sigma$, we can rewrite these equations formally as

$$
\begin{aligned}
& \Delta(\sigma(u) \varphi)-\nabla(\varphi \nabla \sigma(u))=0 \text { in } H^{-1}(\Omega), \\
& \Delta\left(u+\frac{1}{2} \sigma(u) \varphi^{2}\right)-\frac{1}{2} \nabla\left(\varphi^{2} \nabla \sigma(u)\right)=0 \quad \text { in } H^{-1}(\Omega)
\end{aligned}
$$

provided

$$
\begin{gathered}
\varphi \in L^{\infty}(\Omega), \quad u \in H^{1}(\Omega), \\
\sigma(u), \sigma(u) \varphi, \quad \sigma(u) \varphi^{2} \in H^{1}(\Omega) .
\end{gathered}
$$

Equations (3.1), (3.2) mean that

$$
\begin{gathered}
\int_{\Omega}(\nabla(\sigma(u) \varphi) \cdot \nabla \zeta-\varphi \nabla \sigma(u) \cdot \nabla \zeta)=0, \\
\int_{\Omega}\left(\nabla\left(u+\frac{1}{2} \sigma(u) \varphi^{2}\right) \cdot \nabla \zeta-\frac{1}{2} \varphi^{2} \nabla \sigma(u) \cdot \nabla \zeta\right)=0
\end{gathered}
$$

for every $\zeta \in H_{0}^{1}(\Omega)$. Denote by $H_{\Gamma_{D}}^{1}(\Omega)$ the class of all functions in $H^{1}(\Omega)$ such that $\zeta=0$ on $\Gamma_{D}$.

Definition 3.1. A pair $(\varphi, u)$ is called a weak solution of the thermistor problem (1.1), (1.3), (1.4) if (3.3), (3.4) hold; if (3.5), (3.6) hold for any $\zeta \in H_{\Gamma_{D}}^{1}(\Omega)$; and if

$$
u-u^{0}=0 \quad \text { on } \Gamma_{D}, \quad \sigma(u) \varphi-\sigma\left(u^{0}\right) \varphi^{0}=0 \quad \text { on } \Gamma_{D} .
$$

REMARK 3.1. By the trace theorem, all the functions in (3.7) are well defined. The trace of $\varphi$ may not be defined, so we have used the trace of $\sigma(u) \varphi$ instead.

REMARK 3.2. Equations (3.5), (3.6) for all $\zeta \in H_{0}^{1}(\Omega)$ mean the same thing as the equations (3.1), (3.2) (which are a weak form of (1.1), (1.3)). The additional 
freedom of choosing $\zeta$ in the larger class $H_{\Gamma_{D}}^{1}(\Omega)$ accounts for a weak form of the Neumann conditions

$$
\frac{\partial \varphi}{\partial n}=0, \quad \frac{\partial u}{\partial n}=0 \quad \text { on } \partial \Omega \backslash \bar{\Gamma}_{D} .
$$

Theorem 3.1. Assume that $\partial \Omega$ and $\partial \Gamma_{D}$ are piecewise in $C^{1+\delta}$ and that (2.1), (2.2) and (2.6)-(2.8) are satisfied. Then there exists a weak solution of the thermistor problem (1.1), (1.3), (1.4).

Proof. By Lemma 2.2 there exists a sequence $\varepsilon \rightarrow 0$ and functions

$$
\varphi \in L^{\infty}(\Omega), \quad u \in L^{\infty}(\Omega) \cap H^{1}(\Omega), \quad \sigma_{0}, h, \text { and } g \text { in } H^{1}(\Omega)
$$

such that

$$
\begin{array}{ll}
\varphi_{\varepsilon} \rightarrow \varphi & \text { weakly in }\left(L^{\infty}(\Omega)\right)^{*}, \\
u_{\varepsilon} \rightarrow u & \text { weakly in } H^{1}(\Omega) \text { and a.e. in } \Omega, \\
\sigma_{\varepsilon}\left(u_{\varepsilon}\right) \rightarrow \sigma_{0} & \text { weakly in } H^{1}(\Omega) \text { and a.e. in } \Omega, \\
\sigma_{\varepsilon}\left(u_{\varepsilon}\right) \varphi_{\varepsilon} \rightarrow h & \text { weakly in } H^{1}(\Omega) \text { and a.e. in } \Omega, \\
\sigma_{\varepsilon}\left(u_{\varepsilon}\right) \varphi_{\varepsilon}^{2} \rightarrow g & \text { weakly in } H^{1}(\Omega) \text { and a.e. in } \Omega .
\end{array}
$$

Recalling (2.4) we conclude from (3.9), (3.10) that

$$
\sigma_{0}(x)=\sigma(u(x)) \text { a.e. in } \Omega \text {. }
$$

Set

$$
\Omega_{0}=\{x \in \bar{\Omega} ; \sigma(u(x))=0\} \quad(=\{x \in \bar{\Omega} ; u(x) \geq 0\}) .
$$

Then (3.11) implies that

$$
\varphi_{\varepsilon}=\frac{\sigma_{\varepsilon}\left(u_{\varepsilon}\right) \varphi_{\varepsilon}}{\sigma_{\varepsilon}\left(u_{\varepsilon}\right)} \rightarrow \frac{h}{\sigma(u)} \quad \text { a.e. in } \Omega \backslash \Omega_{0} .
$$

On the other hand, from (3.11) and the uniform boundedness of the $\varphi_{\varepsilon}$ we have that $h=0$ a.e. in $\Omega_{0}$, and so $h=\sigma \varphi$ a.e. on $\Omega_{0}$. Thus

$$
h=\sigma(u) \varphi \text { a.e. in } \Omega
$$

and similarly

$$
g=\sigma(u) \varphi^{2} \quad \text { a.e. in } \Omega .
$$

Clearly (by the trace theorem) also

$$
u-u^{0}=0 \quad \text { on } \Omega_{D}, \quad h-\sigma\left(u^{0}\right) \varphi^{0}=0 \quad \text { on } \Gamma_{D} .
$$

To complete the proof of the theorem it remains to show that $(\varphi, u)$ satisfies (3.5), (3.6). These equations of course hold for $\left(\varphi_{\varepsilon}, u_{\varepsilon}\right)$, so that it only remains to justify the passage to the limit. Since $\sigma_{\varepsilon}\left(u_{\varepsilon}\right) \rightarrow \sigma(u)$ and $\varphi_{\varepsilon}^{k} \sigma_{\varepsilon}\left(u_{\varepsilon}\right) \rightarrow \varphi^{k} \sigma(u)$ $(k=1,2)$ weakly in $H^{1}(\Omega)$,

$$
\begin{aligned}
& \int_{\Omega} \nabla \sigma_{\varepsilon}\left(u_{\varepsilon}\right) \cdot \nabla \zeta \rightarrow \int_{\Omega} \nabla \sigma(u) \cdot \nabla \zeta, \\
& \int_{\Omega} \nabla\left(\varphi_{\varepsilon}^{k} \sigma_{\varepsilon}\left(u_{\varepsilon}\right)\right) \cdot \nabla \zeta \rightarrow \int_{\Omega} \nabla\left(\varphi^{k} \sigma(u)\right) \cdot \nabla \zeta
\end{aligned}
$$


as $\varepsilon \rightarrow 0$. Thus it remains to show that

$$
\int_{\Omega} \varphi_{\varepsilon} \nabla \sigma_{\varepsilon}\left(u_{\varepsilon}\right) \cdot \nabla \zeta \rightarrow \int_{\Omega} \varphi \nabla \sigma(u) \cdot \nabla \zeta
$$

and

$$
\int_{\Omega} \varphi_{\varepsilon}^{2} \nabla \sigma_{\varepsilon}\left(u_{\varepsilon}\right) \cdot \nabla \zeta \rightarrow \int_{\Omega} \varphi^{2} \nabla \sigma(u) \cdot \nabla \zeta .
$$

Since $\nabla \sigma_{\varepsilon} \rightarrow \nabla \sigma$ weakly in $L^{2}(\Omega)$ and $\varphi_{\varepsilon} \rightarrow \varphi$ strongly in $L^{2}\left(\Omega \backslash \Omega_{0}\right)$ (since $\varphi_{\varepsilon} \rightarrow \varphi$ a.e. in $\Omega \backslash \Omega_{0}$ and weakly $\left.\left(L^{\infty}(\Omega)\right)^{*}\right)$, we easily find that

$$
\int_{\Omega \backslash \Omega_{0}} \varphi_{\varepsilon} \nabla \sigma_{\varepsilon}\left(u_{\varepsilon}\right) \cdot \nabla \zeta \rightarrow \int_{\Omega \backslash \Omega_{0}} \varphi \nabla \sigma(u) \cdot \nabla \zeta .
$$

Next, choose $\beta=1-\delta(\delta>0)$ such that (2.26) holds. Then

$$
\begin{aligned}
\int_{\Omega_{0}}\left|\varphi_{\varepsilon} \nabla \sigma_{\varepsilon}\left(u_{\varepsilon}\right) \cdot \nabla \zeta\right| & \leq C \int_{\Omega_{0}}\left|\nabla \sigma_{\varepsilon}\left(u_{\varepsilon}\right)\right|=\frac{C}{\beta} \int_{\Omega_{0}} \sigma_{\varepsilon}^{\delta}\left(u_{\varepsilon}\right)\left|\nabla \sigma_{\varepsilon}^{\beta}\left(u_{\varepsilon}\right)\right| \\
& \leq \frac{C}{\beta}\left\|\sigma_{\varepsilon}^{\delta}\left(u_{\varepsilon}\right)\right\|_{L^{2}\left(\Omega_{0}\right)}\left\|\nabla \sigma_{\varepsilon}^{\beta}\left(u_{\varepsilon}\right)\right\|_{L^{2}\left(\Omega_{0}\right)} \\
& \leq C_{1}\left\|\sigma_{\varepsilon}^{\delta}\left(u_{\varepsilon}\right)\right\|_{L^{2}\left(\Omega_{0}\right)}
\end{aligned}
$$

by (2.26). By the Lebesgue dominated convergence theorem, the right-hand side converges to zero as $\varepsilon \rightarrow 0$ since $\sigma_{\varepsilon}\left(u_{\varepsilon}\right) \rightarrow \sigma(u)=0$ a.e. on $\Omega_{0}$, whereas $\left|\sigma_{\varepsilon}\left(u_{\varepsilon}\right)\right| \leq$ $2 M$. Thus

$$
\int_{\Omega_{0}} \varphi_{\varepsilon} \nabla \sigma_{\varepsilon}\left(u_{\varepsilon}\right) \cdot \nabla \zeta \rightarrow 0=\int_{\Omega_{0}} \varphi \nabla \sigma(u) \cdot \nabla \zeta
$$

Combining this with (3.17), the assertion (3.15) follows. The proof of (3.16) is similar.

THEOREM 3.2. The weak solution $(\varphi, u)$ established in Theorem 3.1 satisfies:

$$
\begin{gathered}
\Delta u \leq 0 \quad \text { in } \mathscr{O}^{\prime}(\Omega), \\
u^{0} \leq u \leq 0 \quad \text { a.e. in } \Omega .
\end{gathered}
$$

Proof. The assertion (3.18) follows from $\Delta u_{\varepsilon}=-\sigma_{\varepsilon}\left(u_{\varepsilon}\right)\left|\nabla \varphi_{\varepsilon}\right|^{2} \leq 0$. Since $u_{\varepsilon} \geq u^{0}$ in $\Omega$, also $u \geq u^{0}$ a.e. in $\Omega$. Finally, from the uniform boundedness of the functions $\psi_{\varepsilon}$ defined in (2.15) it follows that

$$
\limsup _{\varepsilon \rightarrow 0} u_{\varepsilon}(x) \leq 0 \quad \forall x \in \Omega,
$$

so that $u \leq 0$ a.e.

4. Additional properties of weak solutions. In this section we specialize to the boundary conditions (1.7) (with $u_{1}<0, u_{2}<0$ ) and derive more specific properties of the weak solution; we shall also prove a uniqueness theorem. Except for the proof of uniqueness we shall not actually need the assumption (2.2). 
For simplicity we choose the $\sigma_{\varepsilon}(s)$ such that $\sigma_{\varepsilon}(s)=\sigma(s)$ if $s \leq \max \left\{u_{1}, u_{2}\right\}$. One can determine uniquely constants $a, b$ such that

$$
\frac{1}{2} \varphi_{i}^{2}+\int_{-1}^{u_{i}} \frac{d t}{\sigma(t)}=a \varphi_{i}+b \quad(i=1,2) .
$$

It then follows that

$$
\psi_{\varepsilon}=a \varphi_{\varepsilon}+b \text { in } \Omega,
$$

since both sides satisfy the same elliptic equation $\operatorname{div}\left(\sigma_{\varepsilon}\left(u_{\varepsilon}\right) w\right)=0$, the same Dirichlet data on $\Gamma_{D}$, and both have zero normal derivatives on $\Gamma_{N}$.

It follows (recalling (2.16)) that

$$
\nabla\left(\tilde{a}_{\varepsilon}\left(\varphi_{\varepsilon}\right) \nabla \varphi_{\varepsilon}\right)=0 \text { in } \Omega
$$

where

$$
\tilde{a}_{\varepsilon}(\varphi)=a_{\varepsilon}\left(-\frac{1}{2} \varphi^{2}+a \varphi+b\right)
$$

and $a_{\varepsilon}$ is defined in (2.20).

Setting

$$
A_{\varepsilon}(s)=\int_{0}^{s} \tilde{a}_{\varepsilon}(t) d t
$$

we deduce that the function

$$
w_{\varepsilon}(x)=A_{\varepsilon}\left(\varphi_{\varepsilon}(x)\right)
$$

satisfies

$$
\begin{gathered}
\nabla^{2} w_{\varepsilon}=0 \quad \text { in } \Omega, \quad w_{\varepsilon}=A_{\varepsilon}\left(\varphi_{i}\right) \quad \text { on } \Gamma_{i} \quad(i=1,2), \\
\frac{\partial w_{\varepsilon}}{\partial n}=0 \quad \text { on } \partial \Omega \backslash \overline{\Gamma_{1} \cup \Gamma_{2} .}
\end{gathered}
$$

In the sequel we shall assume that

$$
b_{0} \equiv \int_{-1}^{0} \frac{d t}{\sigma(t)}<\infty
$$

the case

$$
\int_{-1}^{0} \frac{d t}{\sigma(t)}=\infty
$$

will be discussed in Remark 4.4.

Observe that, as $\varepsilon \rightarrow 0$,

$$
\begin{aligned}
& F_{\varepsilon}(u) \rightarrow\left\{\begin{array}{l}
F(u) \quad \text { if } u<0, \quad\left(F(u)=\int_{-1}^{u} \frac{d t}{\sigma(t)}\right), \\
\infty \quad \text { if } u>0,
\end{array}\right. \\
& F^{\prime}(u)>0 \text { if } u<0, \quad F(0-)=b_{0},
\end{aligned}
$$

where (4.8) was used. Also

$$
\begin{aligned}
& F_{\varepsilon}^{-1}(s) \rightarrow F^{-1}(s) \quad \text { if }-s_{0}<s<\infty,-s_{0}=\int_{-1}^{-\infty} \frac{d t}{\sigma(t)}, \\
& F^{-1}(s)<0, \quad \frac{d}{d s} F^{-1}(s)>0 \quad \text { if }-s_{0}<s<b_{0}, \\
& F^{-1}(s)=0 \text { if } s>b_{0} .
\end{aligned}
$$


Write

$$
-\frac{1}{2} s^{2}+a s+b-b_{0}=-\frac{1}{2}\left(s-s_{1}\right)\left(s-s_{2}\right) .
$$

Clearly, when $s_{1}, s_{2}$ are real, if $s_{1}<s<s_{2}$ then $-s^{2} / 2+a s+b>b_{0}$; and if $s<s_{1}$ or $s>s_{2}$ then $-s^{2} / 2+a s+b<b_{0}$.

It follows that if

$$
s_{1}, s_{2} \text { are real and } s_{1}<s_{2} \text {, }
$$

then the function

$$
\tilde{a}(s)=\sigma\left(F^{-1}\left(-\frac{1}{2} s^{2}+a s+b\right)\right) \quad\left(\tilde{a}(s)=\lim _{\varepsilon \rightarrow 0} \tilde{a}_{\varepsilon}(s)\right)
$$

satisfies

$$
\begin{gathered}
\tilde{a}^{\prime}(s)<0 \quad \text { if } s<s_{1}, \quad \tilde{a}^{\prime}(s)>0 \quad \text { if } s>s_{2}, \\
\tilde{a}(s)=0 \quad \text { if } s_{1} \leq s \leq s_{2} .
\end{gathered}
$$

If $s_{1}=s_{2}$ then (4.15) remains valid, whereas if $s_{1}, s_{2}$ are complex then $s^{2} / 2-$ as $b>b_{0}$ for all $s$ and thus

$$
\tilde{a}(s)>0 \text { if } s_{1}, s_{2} \text { are complex. }
$$

We shall first consider the case (4.13). Then, as $\varepsilon \rightarrow 0$,

$$
A_{\varepsilon}(s) \rightarrow A(s)
$$

uniformly on bounded sets,

$$
\begin{gathered}
A^{\prime}(s)>0 \quad \text { if } s<s_{1} \quad \text { or } \quad s>s_{2}, \\
A(s)=A_{*} \equiv \int_{0}^{s_{1}} \tilde{a}(t) d t \quad \text { if } s_{1}<s<s_{2} .
\end{gathered}
$$

The harmonic function

$$
w_{\varepsilon}(x)=A_{\varepsilon}\left(\varphi_{\varepsilon}(x)\right)=\int_{0}^{\varphi_{\varepsilon}(x)} \tilde{a}_{\varepsilon}(s) d s
$$

then satisfies

$$
w_{\varepsilon} \rightarrow w
$$

uniformly in compact subsets of $\bar{\Omega} \backslash\left(\bar{\Gamma}_{D} \cap \bar{\Gamma}_{N}\right)$, where

$$
\begin{aligned}
& \Delta w=0 \quad \text { in } \Omega \\
& w=A\left(\varphi_{i}\right) \quad \text { on } \Gamma_{i} \quad(i=1,2), \\
& \frac{\partial w}{\partial n}=0 \quad \text { on } \Gamma_{N} .
\end{aligned}
$$

Introduce the inverse function $A^{-1}$ of $A$; clearly,

$$
\begin{aligned}
& \frac{d}{d t} A^{-1}(t)>0 \text { if } t<A_{*} \text { or if } t>A_{*}, \\
& A^{-1}\left(A_{*}\right) \text { is the interval }\left\{s_{1}<s<s_{2}\right\} .
\end{aligned}
$$

From (4.19), (4.20), (4.22) we deduce that

$$
\varphi_{\varepsilon}(x)=A_{\varepsilon}^{-1}\left(w_{\varepsilon}(x)\right) \rightarrow A^{-1}(w(x)) \quad \text { in } \Omega \backslash S,
$$


where

$$
S=\left\{x \in \Omega ; w(x)=A_{*}\right\} .
$$

From (2.15), (4.2) we also deduce that

$$
\begin{aligned}
& u_{\varepsilon}(x) \rightarrow u(x) \quad \text { in } \Omega \backslash S, \\
& u(x) \leq 0,
\end{aligned}
$$

and

$$
-\frac{1}{2} \varphi^{2}+a \varphi+b-b_{0}=\int_{0}^{u} \frac{d s}{\sigma(s)}
$$

further

$$
\nabla w=\sigma(u) \nabla \varphi \quad \text { in } \Omega \backslash S .
$$

The set $S$ is a level surface of the harmonic function $w$, and it is therefore piecewise analytic; in case $N=2, S$ is actually an analytic curve. We are assuming here that

$$
A_{*} \text { lies between the number } A\left(\varphi_{1}\right), A\left(\varphi_{2}\right) \text {; }
$$

otherwise $S$ is empty.

Set

$$
\Omega_{+}=\left\{x \in \Omega, w(x)>A_{*}\right\}, \quad \Omega_{-}=\left\{x \in \Omega, w(x)<A_{*}\right\} ;
$$

each set is a connected open set. Then

$$
\varphi(x)=A^{-1}(w(x)) \quad \text { in } \Omega_{+} \cup \Omega_{-} .
$$

Since $A^{-1}(w(x))$ is continuous in $\bar{\Omega}_{+}$and in $\bar{\Omega}_{-}$with

$$
\lim _{x \rightarrow x_{0}, x \in \Omega_{+}} A^{-1}(w(x))=s_{2}, \quad \lim _{x \rightarrow x_{0}, x \in \Omega_{-}} A^{-1}(w(x))=s_{1}
$$

for any $x_{0} \in S$, it follows that

$$
\begin{gathered}
\varphi \in C^{0}\left(\Omega_{+}\right), \quad \varphi \in C^{0}\left(\Omega_{-}\right) \text {with } \\
\lim _{x \rightarrow x_{0}, x \in \Omega_{+}} \varphi(x)=s_{2}, \quad \lim _{x \rightarrow x_{0}, x \in \Omega_{-}} \varphi(x)=s_{1} \quad \forall x_{0} \in S .
\end{gathered}
$$

Recalling (4.27) we also deduce that

$$
\int_{0}^{u(x)} \frac{d s}{\sigma(s)} \rightarrow 0 \quad \text { if } x \in \Omega \backslash S, \quad x \rightarrow x_{0} \in S,
$$

so that

$$
u(x) \text { is continuous across } S \text {. }
$$

From (4.27) we also deduce that $u<0$ in $\Omega \backslash S$ and $u=0$ on $S$; thus

$$
S \text { is the set }\{x \in \Omega ; \sigma(u(x))=0\} \text {. }
$$

THEOREM 4.1. Assume that $\partial \Omega$ and $\partial \Gamma_{D}$ are piecewise in $C^{1+\delta}$ and that (2.1), (1.7) hold with $u_{1}<0, u_{2}<0$. Then the limit $(\varphi, u)$ of $\left(\varphi_{\varepsilon}, u_{\varepsilon}\right)$ exists and is 
independent of the choice of the family $\sigma_{\varepsilon}$, and it has the following properties:

$\varphi$ and $u$ are related by (4.27),

$u(x)$ and $\sigma(u(x))$ are continuous in $\Omega$,

$\varphi(x)$ is continuous in $\Omega \backslash S$ with limits $s_{2}, s_{1}$ from the respective sides $\Omega_{+}, \Omega_{-}$of $S$,

where

$S$ is the $A_{*}$-level surface of the harmonic function $w$ defined by (4.21),

$$
\begin{gathered}
\sigma(u) \nabla \varphi \in L^{1}(\Omega \backslash S), \\
\nabla u \in L_{\mathrm{loc}}^{\infty}(\Omega),
\end{gathered}
$$

and, finally,

$$
\int_{\Omega \backslash S} \sigma(u) \nabla \varphi \cdot \nabla \zeta=0 \quad \forall \zeta \in H^{1}(\Omega), \quad \zeta=0 \quad \text { on } \Gamma_{1} \cup \Gamma_{2} .
$$

Proof. We have already proved (4.35)-(4.39) ((4.39) follows from (4.28)). From (4.27),

$$
\nabla u=(-\varphi+a) \sigma(u) \nabla \varphi=\left(\frac{s_{1}+s_{2}}{2}-\varphi\right) \nabla w,
$$

and since $\nabla w \in L_{\text {loc }}^{\infty}(\Omega),(4.40)$ follows. It remains to prove $(4.41)$. But this follows from (4.28):

$$
\int_{\Omega \backslash S} \sigma(u) \nabla \varphi \cdot \nabla \zeta=\int_{\Omega \backslash S} \nabla w \cdot \nabla \zeta=\int_{\Omega} \nabla w \cdot \nabla \zeta=\int_{\partial \Omega} \frac{\partial w}{\partial n} \zeta=0 .
$$

REMARK 4.1. From (4.42) we deduce the jump relations

$$
\left[\frac{\partial u}{\partial n}\right]_{S}=-[\varphi]_{S} \frac{\partial w}{\partial n}=-\left[a \varphi \frac{\partial \varphi}{\partial n}\right]_{S}
$$

This implies that equation (1.3) holds in the following sense:

$$
\Delta u+\sigma(u)|\nabla \varphi|^{2} \chi_{\Omega \backslash S}+[\varphi]_{S}(\nabla w \cdot n) \delta_{S}=0
$$

where $\delta_{S}$ is the Dirac function with uniform mass distribution 1 on $S$.

REMARK 4.2. In establishing Theorem 4.1 we have not used condition (2.2).

REMARK 4.3. Theorem 4.1 extends to the case where (4.13) holds with $s_{1}=s_{2}$; in this case $\varphi(x)$ is continuous across $S$. If $s_{1}, s_{2}$ are complex, then (because of (4.16)) the assertions of Theorem 4.1 hold with $S$ the empty set.

REMARK 4.4. So far we have assumed that (4.8) holds. Since $\varphi_{\varepsilon}$ and $\psi_{\varepsilon}$ are uniformly bounded, we also have

$$
\left|\int_{-1}^{u_{\varepsilon}(x)} \frac{d s}{\sigma_{\varepsilon}(s)}\right| \leq C .
$$

If (4.9) holds then the last inequality implies that $u_{\varepsilon}(x) \leq-\delta$, where $\delta$ is a positive constant independent of $\varepsilon$. It follows that for the limiting $(\varphi, u), \sigma(u(x))$ is uniformly positive in $\Omega$. 
From now on we shall assume, in addition to the assumptions of Theorem 4.1, that $\sigma$ satisfies $(2.2)$. Then, by Theorem 3.1, $(\varphi, u)$ is a weak solution, as defined in Sec. 3. Therefore (3.5), (3.6) hold.

We wish to prove (under some assumptions) uniqueness of the weak solution. In general, a weak solution may not be unique. For instance, if $\sigma(u)$ vanishes on a nonempty open set (examples will be given in Sec. 5), then by modifying $\varphi$ in this set we get another weak solution.

Let $(\varphi, \psi)$ be a weak solution of $(1.1),(1.3),(1.7)$. We shall make several assumptions:

$$
\begin{aligned}
& \sigma(u) \text { is continuous in } \Omega, \\
& \text { meas }\{\sigma(u)=0\}=0,
\end{aligned}
$$

and

each component of $\{\sigma(u)>0\}$ is connected to $\Gamma_{D}\left(=\Gamma_{1} \cup \Gamma_{2}\right)$; further, setting

and

$$
\psi=\left\{\begin{array}{l}
\frac{1}{2} \varphi^{2}+\int_{0}^{u} \frac{d s}{\sigma(s)} \quad \text { in }\{\sigma(u) \neq 0\}, \\
\frac{1}{2} \varphi^{2} \quad \text { in } \Omega_{0} \equiv\{\sigma(u)=0\},
\end{array}\right.
$$

$$
\eta=\psi-a \varphi-b
$$

where $a, b$ are constants such that $\eta=0$ on $\Gamma_{D}$ (see (4.1)), we assume that

$$
\eta \in H^{1}(\Omega) \text {. }
$$

TheOREM 4.2. Let the assumptions of Theorem 4.1 and (2.2) hold. Then there exists at most one weak solution of the thermistor problem (1.1), (1.3), (1.7) satisfying (4.44)-(4.46), (4.49).

Of course, the existence of such a solution and additional properties of it were established in Theorem 4.1.

Proof. Since $\sigma \varphi$ and $\eta$ belong to $H^{1}(\Omega)$, the same is true of $\sigma \psi$. One can easily verify that $\sigma \nabla \psi=\sigma \varphi \nabla \varphi+\nabla u$ a.e. in both $\Omega_{1} \equiv\{\sigma(u)>0\}$ and $\Omega_{0}$. Using (3.5), (3.6) we then deduce that $\psi$ satisfies the same equation (3.5) as $\varphi$, and therefore

$$
\int_{\Omega}(\nabla(\sigma(u) \eta) \cdot \nabla \zeta-\eta \nabla \sigma(u) \cdot \nabla \zeta)=0 \quad \forall \zeta \in H_{\Gamma_{D}}^{1}(\Omega) .
$$

Since $\eta=0$ on $\Gamma_{D}$ (in the trace class), we can take $\zeta=\eta$ :

$$
\int_{\Omega}(\nabla(\sigma(u) \eta) \cdot \nabla \eta-\eta \nabla \sigma(u) \cdot \nabla \eta)=0, \quad \text { or } \quad \int_{\Omega} \sigma(u)|\nabla \eta|^{2}=0
$$

Recalling (4.46) we conclude that $\eta=0$ a.e. in $\Omega_{1}$, and consequently, by (4.45), $\eta=0$ a.e. in $\Omega$.

One can now proceed as in the proof of Theorem 4.1, and derive for $\varphi$ a nonlinear elliptic equation: $\nabla(\tilde{a}(\varphi) \nabla \varphi)=0$ with $\tilde{a}(\varphi)$ defined as in (4.14). But then $\varphi$ must coincide with the function $\varphi$ which was obtained in Theorem 4.1. Since $\varphi$ is uniquely determined, also $u$ is uniquely determined. 
REMARK 4.5. Without the assumption (4.49) one can construct infinitely many weak solutions. They are obtained by taking

$$
\psi=a^{\prime} \varphi+b^{\prime}+c \chi_{\Omega^{\prime}}, \quad c \text { an arbitrary constant. }
$$

Here $\Omega^{\prime}$ is a subdomain of $\Omega$ (to be determined such that $\overline{\Omega^{\prime}} \supset \Gamma_{1}, \overline{\Omega^{\prime}} \cap \Gamma_{2}=\varnothing$ ) and $a^{\prime}, b^{\prime}$ are constants determined by boundary conditions similar to (4.1), namely, $a \varphi_{i}+b$ is replaced by $a^{\prime} \varphi+b^{\prime}+c$ for $i=1$ and by $a^{\prime} \varphi+b^{\prime}$ for $i=2$. The function $u$ is defined by (4.47), and $\partial \Omega^{\prime} \cap \Omega$ is the set $\{\sigma(u)=0\}$.

5. Examples. We try a solution, in $\mathbb{R}^{2}$, of the form

$$
\varphi=\alpha \Phi(y)+\beta x, \quad u=U(y) .
$$

Then (1.1), (1.3) become

$$
\begin{gathered}
\left(\sigma(U) \Phi^{\prime}\right)^{\prime}=0, \\
U^{\prime \prime}+\sigma(U)\left(\alpha^{2}\left(\Phi^{\prime}\right)^{2}+\beta^{2}\right)=0,
\end{gathered}
$$

so that

$$
\sigma \Phi^{\prime}=\text { const }=C_{1}
$$

and

$$
U^{\prime \prime}+\sigma(U) \beta^{2}+\frac{\alpha^{2} C_{1}^{2}}{\sigma(U)}=0 \quad \text { in }\{\sigma(U)>0\} .
$$

The last equation can be reduced to

$$
\frac{1}{2} U^{\prime 2}+\int_{0}^{u}\left(\beta^{2} \sigma(s)+\frac{\alpha^{2} C_{1}^{2}}{\sigma(s)}\right) d s=\text { const }=C_{2},
$$

or

$$
U^{\prime}=F\left(U, C_{1}, C_{2}\right) \text {. }
$$

Take for example $\alpha=0, \beta=1$ and assume that

$$
\sigma(s)= \begin{cases}c|s|^{\gamma} & \text { if } s<0 \\ 0 & \text { if } s>0\end{cases}
$$

where $0<\gamma<1$ and $c$ is a positive constant. Then a solution to (5.4) is given by

$$
u_{0}(y)= \begin{cases}-y^{\delta} & \text { if } y>0, \\ 0 & \text { if }-\mu<y<0, \\ -(-\mu-y)^{\delta} & \text { if } y<-\mu\end{cases}
$$

for any $\mu>0$, provided

$$
\delta=\frac{2}{1-\gamma}, \quad c=\delta(\delta-1) .
$$

One may perceive $\left(x, u_{0}(y)\right)$ as a weak solution in a rectangle $\Omega$, with boundary conditions

$$
\begin{aligned}
& \frac{\partial \varphi}{\partial n}=0 \quad \text { on the horizontal edges of } \partial \Omega, \\
& \frac{\partial u}{\partial n}=0 \text { on the vertical edges of } \partial \Omega,
\end{aligned}
$$


and $\varphi=\varphi_{0}, u=u_{0}$ (suitable functions) on the remaining edges.

In the above example

$$
\text { the set }\{\sigma(u)=0\} \text { is a strip }\{-\mu<y<0\} \text {; }
$$

the boundary conditions are of course not of the form (1.7) (or even (1.4)).

In case $\alpha \neq 0$, for the corresponding solution of $(5.2)$ the set $\{\sigma(u)=0\}$ has measure zero, in general.

Let

$$
f(z)=f_{1}(x, y)+i f_{2}(x, y) \quad(z=x+i y)
$$

be any holomorphic function. It was observed by Howison [8] that solutions to the thermistor problem are invariant under conformal mappings of the independent variable. Thus, in particular, the pair

$$
\varphi=f_{1}(x, y), \quad u=u_{0}\left(f_{2}(x, y)\right),
$$

where $u_{0}$ is defined by $(5.8)$, is a solution of the thermistor problem, and $\{\sigma(u)=0\}$ has nonempty interior.

Acknowledgment. The first author is supported by Alfred P. Sloan Doctoral Dissertation Fellowship No. DD-318. The second author is partially supported by the National Science Foundation Grant DMS-86-12880.

\section{REFERENCES}

[1] J. Bass, Thermoelasticity, McGraw-Hill Encyclopedia of Physics (S. P. Parker, ed.), McGraw-Hill, New York, 1982

[2] G. Cimatti and G. Prodi, Existence results for a nonlinear elliptic system modelling a temperature dependent electrical resistor, Ann. Math. Pure Appl. 152, 227-236 (1988)

[3] G. Cimatti, $A$ bound for the temperature in the thermistor problem, IMA J. Appl. Math. 40, 15-22 (1988)

[4] _ Remark on existence and uniqueness for the thermistor problem under mixed boundary conditions, Quart. Appl. Math. 47, 117-121 (1989)

[5] H. Diesselhorst, Ueber das Probleme eines elektrisch erwärmten Leiters, Ann. Physics 1, 312-325 (1900)

[6] D. Gilbarg and N. Trudinger, Elliptic Partial Differential Equations of Second Order, 2nd ed., Springer-Verlag, Berlin, 1983

[7] S. Howison, $A$ note on the thermistor problem in two space dimensions, Quart. Appl. Math. 47, 509-512 (1989)

[8] S. D. Howison, J. F. Rodrigues, and M. Shillor, Existence results for the problems of Joule heating of a resistor, to appear

[9] F. J. Hyde, Thermistors, Iliffe Books, London, 1971

[10] J. F. Llewellyn, The Physics of Electrical Contacts, Clarendon Press, Oxford, 1957

[11] J. M. Young, Steady state Joule heating with temperature dependent conductivities, Appl. Sci. Res. 43, 55-65 (1986) 